\title{
Iterative methods for hierarchical common fixed point problems and variational inequalities
}

\author{
D R Sahu', Shin Min Kang ${ }^{2 *}$ and Vidya Sagar ${ }^{1}$
}

\section{"Correspondence:} smkang@gnu.ac.kr

${ }^{2}$ Department of Mathematics and

RINS, Gyeongsang National

University, Jinju, 660-701, Korea

Full list of author information is

available at the end of the article

\begin{abstract}
The purpose of this paper is to deal with the problem of finding hierarchically a common fixed point of a sequence of nearly nonexpansive self-mappings defined on a closed convex subset of a real Hilbert space which is also a solution of some particular variational inequality problem. We introduce two explicit iterative schemes and establish strong convergence results for sequences generated iteratively by the explicit schemes under suitable conditions. Our strong convergence results include the previous results as special cases, and can be viewed as an improvement and refinement of several corresponding known results for hierarchical variational inequality problems.
\end{abstract}

MSC: 47J20; 49J40

Keywords: hierarchical variational inequality; metric projection mapping; nonexpansive mapping; sequence of nearly nonexpansive mappings

\section{Introduction}

Variational inequality problems were initially studied by Stampacchia [1] in 1964. Since then, many kinds of variational inequalities have been extended and generalized in several directions using novel and innovative techniques (see [2-5] and the references therein).

The classical variational inequality problem or the Stampacchia variational inequality problem in a Hilbert space is defined as follows:

Let $C$ be a nonempty closed convex subset of a real Hilbert space $H$, and let $T: C \rightarrow H$ be a nonlinear mapping. Then the classical variational inequality problem is a problem of finding $x^{*} \in C$ such that

$$
\left\langle T x^{*}, y-x^{*}\right\rangle \geq 0 \quad \text { for all } y \in C \text {. }
$$

Problem (1.1) is denoted by $\operatorname{VI}(C, T)$. A point $x^{*} \in C$ is a solution of $\operatorname{VI}(C, T)$ if and only if $x^{*}$ is a fixed point of $P_{C}(I-\lambda T)$, where $\lambda>0$ is a constant, $I$ is the identity mapping from $C$ into itself, and $P_{C}$ is the metric projection from $H$ onto a closed convex subset $C$ of $H$. The set of solutions of (1.1) is denoted by $\Omega(C, T)$, that is,

$$
\Omega(C, T)=\left\{x^{*} \in C:\left\langle T x^{*}, y-x^{*}\right\rangle \geq 0 \text { for all } y \in C\right\} .
$$

O\#CPRSahu et al.: licensee Springer. This is an Open Access article distributed under the terms of the Creative Commons Attribution License (http://creativecommons.org/licenses/by/2.0), which permits unrestricted use, distribution, and reproduction in any medium, provided the original work is properly cited. 
The variational inequality $\operatorname{VI}(C, T)$ is called a monotone variational inequality if the operator $T$ is a monotone operator. The problem of kind (1.1) is connected with the convex minimization problem, complementarity problem, saddle point problem, fixed point problem, Nash equilibrium problem, the problem of finding a point $x \in H$ satisfying $T x=0$ and so on, and it has several applications in different branches of natural sciences, social sciences, management and engineering (see [6-11] and the references therein). In this context, we discuss the variational inequality problem over the set of fixed points of a mapping which is known as a hierarchical variational inequality problem or a hierarchical fixed point problem.

The hierarchical variational inequality problem over the set of fixed points of a nonexpansive mapping is defined as follows:

Let $C$ be a nonempty closed convex subset of a real Hilbert space $H$, and let $T, S: C \rightarrow C$ be two nonexpansive mappings. Then the hierarchical variational inequality problem is given as follows:

$$
\text { Find } x^{*} \in F(S) \text { such that }\left\langle(I-T) x^{*}, y-x^{*}\right\rangle \geq 0 \text { for all } y \in F(S) \text {. }
$$

Problem (1.2) is denoted by $\mathrm{VI}_{F(S)}(C, T)$. The set of solutions of (1.2) is denoted by $\Omega_{F(S)}(C, T)$, that is,

$$
\Omega_{F(S)}(C, T)=\left\{x^{*} \in F(S):\left\{(I-T) x^{*}, y-x^{*}\right\rangle \geq 0 \text { for all } y \in F(S)\right\} .
$$

It is easy to observe that $\mathrm{VI}_{F(S)}(C, T)$ is equivalent to the fixed point problem $x^{*}=P_{F(S)} T x^{*}$, that is, $x^{*}$ is a fixed point of the nonexpansive mapping $P_{F(S)} T$. Of course, if $T=I$, then the set of solutions of $\mathrm{VI}_{F(S)}(C, T)$ is $\Omega_{F(S)}(C, T)=F(S)$.

Firstly, Moudafi and Maingé [12] introduced an implicit iterative algorithm to solve problem (1.2) and proved weak and strong convergence results, and after that, many iterative methods have been developed for solving hierarchical problem (1.2) by several authors (see, e.g., [13-17]).

Very recently, Gu et al. [18] motivated and inspired by the results of Marino and Xu [15], Yao et al. [17] introduced and studied two iterative schemes for solving hierarchical variational inequality problem and proved the corresponding strong convergence results for the generated sequences in the context of a countable family of nonexpansive mappings under suitable conditions on parameters.

In this paper, inspired by Gu et al. [18] and Sahu et al. [19], we introduce two explicit iterative schemes which generate sequences via iterative algorithms. We prove that the generated sequences converge strongly to the unique solutions of particular variational inequality problems defined over the set of common fixed points of a sequence of nearly nonexpansive mappings. Our results, in one sense, extend the results of Gu et al. [18] to the sequence of nonexpansive mappings and, in another sense, to the sequence of nearly nonexpansive mappings, which is a wider class of sequence of nonexpansive mappings. Our results also generalize the results of Cianciaruso et al. [13], Yao et al. [17], Moudafi [20], Xu [21] and many other related works.

\section{Preliminaries}

Let $C$ be a nonempty subset of a real Hilbert space $H$ with the inner product $\langle\cdot, \cdot\rangle$ and the norm $\|\cdot\|$, respectively. A mapping $T: C \rightarrow H$ is called 
(1) monotone if

$$
\langle T x-T y, x-y\rangle \geq 0 \quad \text { for all } x, y \in C,
$$

(2) $\eta$-strongly monotone if there exists a positive real number $\eta$ such that

$$
\langle T x-T y, x-y\rangle \geq \eta\|x-y\|^{2} \quad \text { for all } x, y \in C,
$$

(3) $k$-Lipschitzian if there exists a constant $k>0$ such that

$$
\|T x-T y\| \leq k\|x-y\| \quad \text { for all } x, y \in C
$$

(4) $\rho$-contraction if there exists a constant $\rho \in(0,1)$ such that

$$
\|T x-T y\| \leq \rho\|x-y\| \quad \text { for all } x, y \in C,
$$

(5) nonexpansive if

$$
\|T x-T y\| \leq\|x-y\| \quad \text { for all } x, y \in C,
$$

(6) nearly nonexpansive $[22,23]$ with respect to a fixed sequence $\left\{a_{n}\right\}$ in $[0, \infty)$ with

$$
a_{n} \rightarrow 0 \text { if }
$$

$$
\left\|T^{n} x-T^{n} y\right\| \leq\|x-y\|+a_{n} \quad \text { for all } x, y \in C \text { and } n \in \mathbb{N}
$$

Throughout this paper, we denote by $I$ the identity mapping of $H$. Also, we denote by $\rightarrow$ and $\rightarrow$ the strong convergence and weak convergence, respectively. The symbol $\mathbb{N}$ stands for the set of all natural numbers and $\omega_{w}\left(\left\{x_{n}\right\}\right)$ denotes the set of all weak subsequential limits of $\left\{x_{n}\right\}$.

Let $C$ be a nonempty closed convex subset of $H$. Then, for any $x \in H$, there exists a unique nearest point in $C$, denoted by $P_{C}(x)$, such that

$$
\left\|x-P_{C}(x)\right\|=\inf \|x-y\| \quad \text { for all } y \in C
$$

The mapping $P_{C}$ is called the metric projection from $H$ onto $C$.

It is observed that $P_{C}$ is a nonexpansive and monotone mapping from $H$ onto $C$ (see Agarwal et al. [22] for other properties of projection operators).

Let $C$ be a nonempty subset of a real Hilbert space $H$, and let $S_{1}, S_{2}: C \rightarrow H$ be two mappings. We denote by $\mathcal{B}(C)$ the collection of all bounded subsets of $C$. The deviation between $S_{1}$ and $S_{2}$ on $B \in \mathcal{B}(C)$ [24], denoted by $\left\|S_{1}-S_{2}\right\|_{\infty, B}$, is defined by

$$
\left\|S_{1}-S_{2}\right\|_{\infty, B}=\sup \left\{\left\|S_{1}(x)-S_{2}(x)\right\|: x \in B\right\} .
$$

In what follows, we shall make use of the following lemmas and proposition.

Lemma 2.1 ([11]) Let $C$ be a nonempty closed convex subset of a real Hilbert space $H$. If $x \in H$ and $y \in C$, then $y=P_{C}(x)$ if and only if the following inequality holds:

$$
\langle x-y, z-y\rangle \leq 0 \quad \text { for all } z \in C \text {. }
$$


Lemma 2.2 ([25]) Let $f: C \rightarrow H$ be a $\lambda$-contraction mapping and $T: C \rightarrow C$ be nonexpansive. Then the following hold:

(a) The mapping $I-f$ is $(1-\lambda)$-strongly monotone, i.e.,

$$
\langle x-y,(I-f) x-(I-f) y| \geq(1-\lambda)\|x-y\|^{2} \quad \text { for all } x, y \in C .
$$

(b) The mapping I-T is monotone, i.e.,

$$
\langle x-y,(I-T) x-(I-T) y\rangle \geq 0 \quad \text { for all } x, y \in C .
$$

Lemma 2.3 ([22]) Let $T$ be a nonexpansive self-mapping of a nonempty closed convex subset $C$ of a real Hilbert space $H$. Then $I-T$ is demiclosed at zero, i.e., if $\left\{x_{n}\right\}$ is a sequence in $C$ weakly converging to some $x \in C$ and the sequence $\left\{(I-T) x_{n}\right\}$ strongly converges to 0 , then $x \in F(T)$.

Lemma 2.4 ([26]) Let $\left\{t_{n}\right\}$ and $\left\{d_{n}\right\}$ be the sequences of nonnegative real numbers such that

$$
t_{n+1} \leq\left(1-b_{n}\right) t_{n}+c_{n}+d_{n} \quad \text { for all } n \in \mathbb{N}
$$

where $\left\{b_{n}\right\}$ is a real number sequence in $(0,1)$ and $\left\{c_{n}\right\}$ is a real number sequence. Assume that the following conditions hold:

(i) $\sum_{n=1}^{\infty} d_{n}<\infty$;

(ii) $\sum_{n=1}^{\infty} b_{n}=\infty$ and $\lim \sup _{n \rightarrow \infty} \frac{c_{n}}{b_{n}} \leq 0$.

Then $\lim _{n \rightarrow \infty} t_{n}=0$.

Lemma 2.5 ([24,27]) Let $C$ be a nonempty closed convex subset of a real Hilbert space $H$ and $t_{i}>0(i=1,2,3, \ldots, N)$ such that $\sum_{i=1}^{N} t_{i}=1$. Let $T_{1}, T_{2}, T_{3}, \ldots, T_{N}: C \rightarrow C$ be nonexpansive mappings with $\bigcap_{i=1}^{N} F\left(T_{i}\right) \neq \emptyset$ and let $T=\sum_{i=1}^{N} t_{i} T_{i}$. Then $T$ is nonexpansive from $C$ into itself and $F(T)=\bigcap_{i=1}^{N} F\left(T_{i}\right)$.

Let $C$ be a nonempty subset of a real Hilbert space $H$. Let $\mathcal{T}:=\left\{T_{n}\right\}_{n=1}^{\infty}$ be a sequence of mappings from $C$ into itself. We denote by $F(\mathcal{T})$ the set of common fixed points of the sequence $\mathcal{T}$, that is, $F(\mathcal{T})=\bigcap_{n=1}^{\infty} F\left(T_{n}\right)$. Fix a sequence $\left\{a_{n}\right\}$ in $[0, \infty)$ with $a_{n} \rightarrow 0$, and let $\left\{T_{n}\right\}$ be a sequence of mappings from $C$ into $H$. Then the sequence $\left\{T_{n}\right\}$ is called a sequence of nearly nonexpansive mappings [19] with respect to a sequence $\left\{a_{n}\right\}$ if

$$
\left\|T_{n} x-T_{n} y\right\| \leq\|x-y\|+a_{n} \quad \text { for all } x, y \in C \text { and } n \in \mathbb{N} .
$$

One can observe that the sequence of nonexpansive mappings is essentially a sequence of nearly nonexpansive mappings.

We now introduce the following:

Let $C$ be a nonempty closed convex subset of a Hilbert space $H$. Let $\mathcal{T}=\left\{T_{n}\right\}_{n=1}^{\infty}$ be a sequence of nearly nonexpansive mappings from $C$ into itself with sequence $\left\{a_{n}\right\}$ such that $\bigcap_{n=1}^{\infty} F\left(T_{n}\right) \neq \emptyset$. Let $T: C \rightarrow C$ be a mapping such that $T x=\lim _{n \rightarrow \infty} T_{n} x$ for all $x \in C$ with $F(T)=\bigcap_{n=1}^{\infty} F\left(T_{n}\right)$. Then $\left\{T_{n}\right\}$ is said to satisfy condition $(G)$ if for each sequence $\left\{x_{n}\right\}$ in $C$ with $x_{n} \rightarrow w$ and $x_{n}-T_{i} x_{n} \rightarrow 0$ for all $i \in \mathbb{N}$ imply $w \in F(T)$. 
Proposition 2.1 Let $C$ be a nonempty closed convex subset of a real Hilbert space H. Let $\left\{T_{n}\right\}$ be a sequence of nonexpansive mappings from $C$ into itself. Then $\left\{T_{n}\right\}$ satisfies condi$\operatorname{tion}(G)$.

Proposition 2.2 Let $C$ be a nonempty closed convex subset of a real Hilbert space H. Let $\mathcal{T}=\left\{T_{n}\right\}_{n=1}^{\infty}$ be a sequence of nearly nonexpansive mappings from $C$ into itself with sequence $\left\{a_{n}\right\}$ such that $\bigcap_{n=1}^{\infty} F\left(T_{n}\right) \neq \emptyset$. Then

$$
\left\|T_{n} x-x\right\|^{2} \leq 2\left\langle x-T_{n} x, x-\tilde{x}\right\rangle+\left(a_{n}+2\|x-\tilde{x}\|\right) a_{n}
$$

for all $x \in C$ and $\tilde{x} \in \bigcap_{n=1}^{\infty} F\left(T_{n}\right)$.

Proof Let $x \in C$ and $\tilde{x} \in \bigcap_{n=1}^{\infty} F\left(T_{n}\right)$. Then

$$
\left\|T_{n} x-T_{n} \tilde{x}\right\|^{2} \leq\left(\|x-\tilde{x}\|+a_{n}\right)^{2}=\|x-\tilde{x}\|^{2}+\left(a_{n}+2\|x-\tilde{x}\|\right) a_{n} .
$$

Since $\tilde{x} \in \bigcap_{n=1}^{\infty} F\left(T_{n}\right)$, we have

$$
\begin{aligned}
\| x & -\tilde{x} \|^{2}+\left(a_{n}+2\|x-\tilde{x}\|\right) a_{n} \\
& \geq\left\|T_{n} x-\tilde{x}\right\|^{2}=\left\|\left(T_{n} x-x\right)+(x-\tilde{x})\right\|^{2} \\
& =\left\|T_{n} x-x\right\|^{2}+\|x-\tilde{x}\|^{2}+2\left\langle T_{n} x-x, x-\tilde{x}\right\rangle .
\end{aligned}
$$

Therefore,

$$
\left\|T_{n} x-x\right\|^{2} \leq 2\left\langle x-T_{n} x, x-\tilde{x}\right\rangle+\left(a_{n}+2\|x-\tilde{x}\|\right) a_{n} .
$$

Proposition 2.3 Let C be a nonempty closed convex subset of a real Hilbert space H. Let $\mathcal{T}=\left\{T_{n}\right\}_{n=1}^{\infty}$ be a sequence of nearly nonexpansive mappings from $C$ into itself with sequence $\left\{a_{n}\right\}$. Then

$$
\left\langle\left(I-T_{n}\right) x-\left(I-T_{n}\right) y, x-y\right\rangle \geq-a_{n}\|x-y\| \quad \text { for all } x, y \in C \text { and } n \in \mathbb{N} .
$$

Proof Let $x, y \in C$. Then

$$
\begin{aligned}
& \left\langle\left(I-T_{n}\right) x-\left(I-T_{n}\right) y, x-y\right\rangle \\
& \quad=\|x-y\|^{2}-\left\langle T_{n} x-T_{n} y, x-y\right\rangle \\
& \quad \geq\|x-y\|^{2}-\left\|T_{n} x-T_{n} y\right\|\|x-y\| \\
& \quad \geq\|x-y\|^{2}-\left(\|x-y\|+a_{n}\right)\|x-y\|=-a_{n}\|x-y\| .
\end{aligned}
$$

\section{Main result}

Theorem 3.1 Let $C$ be a nonempty closed convex subset of a real Hilbert space $H$. Let $f: C \rightarrow H$ be a $\lambda$-contraction, and let $\left\{S_{n}\right\}$ be a sequence of nonexpansive mappings from $C$ into itself. Let $S$ be a nonexpansive mapping from $C$ into itself such that $\lim _{n \rightarrow \infty} S_{n} x=S x$ for all $x \in C$. Let $\mathcal{T}=\left\{T_{n}\right\}_{n=1}^{\infty}$ be a sequence of uniformly continuous nearly nonexpansive 
mappings from $C$ into itself with sequence $\left\{a_{n}\right\}$ such that $F(\mathcal{T}) \neq \emptyset$. Let $T$ be a mapping from $C$ into itself defined by $T x=\lim _{n \rightarrow \infty} T_{n} x$ for all $x \in C$. Suppose that $F(T)=\bigcap_{n=1}^{\infty} F\left(T_{n}\right)$. For arbitrary $x_{1} \in C$, consider the sequence $\left\{x_{n}\right\}$ generated by the following iterative process:

$$
\left\{\begin{array}{l}
x_{1} \in C, \\
y_{n}=\left(1-\beta_{n}\right) x_{n}+\beta_{n} S_{n} x_{n}, \\
x_{n+1}=P_{C}\left[\alpha_{n} f\left(x_{n}\right)+\sum_{i=1}^{n}\left(\alpha_{i-1}-\alpha_{i}\right) T_{i} y_{n}\right]
\end{array}\right.
$$

for all $n \in \mathbb{N}$, where $\alpha_{0}=1,\left\{\alpha_{n}\right\}$ is a strictly decreasing sequence in $(0,1)$ and $\left\{\beta_{n}\right\}$ is a sequence in $(0,1)$ satisfying the conditions:

(i) $\lim _{n \rightarrow \infty} \alpha_{n}=0, \sum_{n=1}^{\infty} \alpha_{n}=\infty$;

(ii) $\sum_{n=1}^{\infty}\left(\alpha_{n-1}-\alpha_{n}\right)<\infty, \sum_{n=1}^{\infty}\left|\beta_{n-1}-\beta_{n}\right|<\infty$ and $\lim _{n \rightarrow \infty} \frac{\beta_{n}}{\alpha_{n}}=\tau \in(0, \infty)$;

(iii) $\lim _{n \rightarrow \infty} \frac{\left(\left(\alpha_{n-1}-\alpha_{n}\right)+\left|\beta_{n}-\beta_{n-1}\right|\right)}{\alpha_{n} \beta_{n}}=0$;

(iv) there exists a constant $N>0$ such that $\frac{1}{\alpha_{n}}\left|\frac{1}{\beta_{n}}-\frac{1}{\beta_{n-1}}\right| \leq N$;

(v) $\lim _{n \rightarrow \infty} \frac{\gamma_{n}}{\beta_{n}}=0$, where $\gamma_{n}:=\sum_{i=1}^{n}\left(\alpha_{i-1}-\alpha_{i}\right) a_{i}$, and either $\sum_{n=1}^{\infty}\left\|S_{n+1}-S_{n}\right\|_{\infty, B}<\infty$ or $\lim _{n \rightarrow \infty} \frac{\left\|S_{n+1}-S_{n}\right\|_{\infty, B}}{\alpha_{n}}=0$ for each $B \in \mathcal{B}(C)$.

Then the sequence $\left\{x_{n}\right\}$ converges strongly to a point $x^{*} \in \bigcap_{n=1}^{\infty} F\left(T_{n}\right)$, which is the unique solution of the following variational inequality:

$$
\left\langle\frac{1}{\tau}(I-f) x^{*}+(I-S) x^{*}, x-x^{*}\right\rangle \geq 0 \quad \text { for all } x \in \bigcap_{n=1}^{\infty} F\left(T_{n}\right) .
$$

Proof It was proved in [17] that variational inequality problem (3.2) has the unique solution. Let $p \in \bigcap_{n=1}^{\infty} F\left(T_{n}\right)$. We now break the proof into the following steps.

Step 1. $\left\{x_{n}\right\}$ is bounded.

From (3.1), we have

$$
\begin{aligned}
\left\|y_{n}-p\right\| & =\left\|\left(1-\beta_{n}\right) x_{n}+\beta_{n} S_{n} x_{n}-p\right\| \\
& \leq\left(1-\beta_{n}\right)\left\|x_{n}-p\right\|+\beta_{n}\left(\left\|S_{n} x_{n}-S_{n} p\right\|+\left\|S_{n} p-p\right\|\right) \\
& \leq\left\|x_{n}-p\right\|+\beta_{n}\left\|S_{n} p-p\right\| .
\end{aligned}
$$

It follows that

$$
\begin{aligned}
& \left\|x_{n+1}-p\right\| \\
& \quad=\left\|P_{C}\left[\alpha_{n} f\left(x_{n}\right)+\sum_{i=1}^{n}\left(\alpha_{i-1}-\alpha_{i}\right) T_{i} y_{n}\right]-P_{C}(p)\right\| \\
& \leq\left\|\alpha_{n} f\left(x_{n}\right)+\sum_{i=1}^{n}\left(\alpha_{i-1}-\alpha_{i}\right) T_{i} y_{n}-p\right\| \\
& \quad=\left\|\alpha_{n}\left(f\left(x_{n}\right)-p\right)+\sum_{i=1}^{n}\left(\alpha_{i-1}-\alpha_{i}\right)\left(T_{i} y_{n}-p\right)\right\| \\
& \quad \leq \alpha_{n}\left(\left\|f\left(x_{n}\right)-f(p)\right\|+\|f(p)-p\|\right)+\sum_{i=1}^{n}\left(\alpha_{i-1}-\alpha_{i}\right)\left\|T_{i} y_{n}-T_{i} p\right\| \\
& \quad \leq \alpha_{n} \lambda\left\|x_{n}-p\right\|+\alpha_{n}\|f(p)-p\|+\sum_{i=1}^{n}\left(\alpha_{i-1}-\alpha_{i}\right)\left(\left\|y_{n}-p\right\|+a_{i}\right)
\end{aligned}
$$




$$
\begin{aligned}
\leq & \alpha_{n} \lambda\left\|x_{n}-p\right\|+\alpha_{n}\|f(p)-p\| \\
& +\left(1-\alpha_{n}\right)\left(\left\|x_{n}-p\right\|+\beta_{n}\left\|S_{n} p-p\right\|\right)+\gamma_{n} \\
\leq & {\left[1-\alpha_{n}(1-\lambda)\right]\left\|x_{n}-p\right\|+\alpha_{n}\|f(p)-p\|+\beta_{n}\left\|S_{n} p-p\right\|+\gamma_{n} . }
\end{aligned}
$$

Note that $\lim _{n \rightarrow \infty} \frac{\beta_{n}}{\alpha_{n}}=\tau \in(0, \infty)$ and $\lim _{n \rightarrow \infty} \frac{\gamma_{n}}{\beta_{n}}=0$, so there exists a constant $K>0$ such that

$$
\frac{\alpha_{n}\|f(p)-p\|+\beta_{n}\left\|S_{n} p-p\right\|+\gamma_{n}}{\alpha_{n}} \leq K \quad \text { for all } n \in \mathbb{N} .
$$

Thus, we have

$$
\begin{aligned}
\left\|x_{n+1}-p\right\| & \leq\left(1-\alpha_{n}(1-\lambda)\right)\left\|x_{n}-p\right\|+\alpha_{n} K \\
& \leq \max \left\{\left\|x_{n}-p\right\|, \frac{K}{1-\lambda}\right\} \quad \text { for all } n \in \mathbb{N}
\end{aligned}
$$

Hence $\left\{x_{n}\right\}$ is bounded. So, $\left\{f\left(x_{n}\right)\right\},\left\{y_{n}\right\},\left\{T_{i} x_{n}\right\}$ and $\left\{T_{i} y_{n}\right\}$ are bounded.

Step 2. $\left\|x_{n+1}-x_{n}\right\| \rightarrow 0$ as $n \rightarrow \infty$.

Set $u_{n}:=\alpha_{n} f\left(x_{n}\right)+\sum_{i=1}^{n}\left(\alpha_{i-1}-\alpha_{i}\right) T_{i} y_{n}$ for all $n \in \mathbb{N}$. Set $M:=\sup _{n>1}\left\{\left\|f\left(x_{n-1}\right)\right\|+\left\|T_{n} y_{n-1}\right\|+\right.$ $\left.\left\|S_{n-1} x_{n-1}\right\|+\left\|x_{n-1}\right\|\right\}$. From (3.1) we have

$$
\begin{aligned}
\left\|x_{n+1}-x_{n}\right\| & \\
= & \left\|P_{C}\left(u_{n}\right)-P_{C}\left(u_{n-1}\right)\right\| \\
\leq & \left\|u_{n}-u_{n-1}\right\| \\
= & \| \alpha_{n}\left(f\left(x_{n}\right)-f\left(x_{n-1}\right)\right)+\left(\alpha_{n}-\alpha_{n-1}\right) f\left(x_{n-1}\right) \\
& +\sum_{i=1}^{n}\left(\alpha_{i-1}-\alpha_{i}\right)\left(T_{i} y_{n}-T_{i} y_{n-1}\right)+\left(\alpha_{n-1}-\alpha_{n}\right) T_{n} y_{n-1} \| \\
\leq & \alpha_{n}\left\|f\left(x_{n}\right)-f\left(x_{n-1}\right)\right\|+\sum_{i=1}^{n}\left(\alpha_{i-1}-\alpha_{i}\right)\left(\left\|y_{n}-y_{n-1}\right\|+a_{i}\right) \\
& +\left(\alpha_{n-1}-\alpha_{n}\right)\left(\left\|f\left(x_{n-1}\right)\right\|+\left\|T_{n} y_{n-1}\right\|\right) \\
\leq & \alpha_{n} \lambda\left\|x_{n}-x_{n-1}\right\|+\sum_{i=1}^{n}\left(\alpha_{i-1}-\alpha_{i}\right)\left\|y_{n}-y_{n-1}\right\| \\
& +\left(\alpha_{n-1}-\alpha_{n}\right)\left(\left\|f\left(x_{n-1}\right)\right\|+\left\|T_{n} y_{n-1}\right\|\right)+\gamma_{n} \\
\leq & \alpha_{n} \lambda\left\|x_{n}-x_{n-1}\right\|+\left(1-\alpha_{n}\right)\left\|y_{n}-y_{n-1}\right\| \\
& +\left(\alpha_{n-1}-\alpha_{n}\right) M+\gamma_{n} .
\end{aligned}
$$

Set $B:=\left\{x_{n}\right\}$. Now, from (3.1) we have

$$
\begin{aligned}
& \left\|y_{n}-y_{n-1}\right\| \\
& \quad=\left\|\left(\left(1-\beta_{n}\right) x_{n}+\beta_{n} S_{n} x_{n}\right)-\left(\left(1-\beta_{n-1}\right) x_{n-1}+\beta_{n-1} S_{n-1} x_{n-1}\right)\right\|
\end{aligned}
$$




$$
\begin{aligned}
= & \|\left(1-\beta_{n}\right)\left(x_{n}-x_{n-1}\right)+\left(\beta_{n-1}-\beta_{n}\right) x_{n-1}+\beta_{n}\left(S_{n} x_{n}-S_{n} x_{n-1}\right) \\
& +\beta_{n}\left(S_{n} x_{n-1}-S_{n-1} x_{n-1}\right)+\left(\beta_{n}-\beta_{n-1}\right) S_{n-1} x_{n-1} \| \\
\leq & \|\left(1-\beta_{n}\right)\left(x_{n}-x_{n-1}\right)+\left(\beta_{n-1}-\beta_{n}\right) x_{n-1}+\beta_{n}\left(S_{n} x_{n}-S_{n} x_{n-1}\right) \\
& +\left(\beta_{n}-\beta_{n-1}\right) S_{n-1} x_{n-1}\left\|+\beta_{n}\right\| S_{n}-S_{n-1} \|_{\infty, B} \\
\leq & \left\|x_{n}-x_{n-1}\right\|+\left|\beta_{n}-\beta_{n-1}\right| M+\beta_{n}\left\|S_{n}-S_{n-1}\right\|_{\infty, B} .
\end{aligned}
$$

Now, using (3.4) in (3.3), we obtain that

$$
\begin{aligned}
\| x_{n+1} & -x_{n} \| \\
\leq & \left\|u_{n}-u_{n-1}\right\| \\
\leq & \alpha_{n} \lambda\left\|x_{n}-x_{n-1}\right\|+\left(1-\alpha_{n}\right)\left[\left\|x_{n}-x_{n-1}\right\|\right. \\
& \left.+\left|\beta_{n}-\beta_{n-1}\right| M+\beta_{n}\left\|S_{n}-S_{n-1}\right\|_{\infty, B}\right]+\left(\alpha_{n-1}-\alpha_{n}\right) M+\gamma_{n} \\
\leq & \left(1-\alpha_{n}(1-\lambda)\right)\left\|x_{n}-x_{n-1}\right\|+M\left[\left(\alpha_{n-1}-\alpha_{n}\right)+\left|\beta_{n}-\beta_{n-1}\right|\right] \\
& +\left(1-\alpha_{n}\right) \beta_{n}\left\|S_{n}-S_{n-1}\right\|_{\infty, B}+\gamma_{n} \\
\leq & \left(1-\alpha_{n}(1-\lambda)\right)\left\|x_{n}-x_{n-1}\right\|+M\left[\left(\alpha_{n-1}-\alpha_{n}\right)+\left|\beta_{n}-\beta_{n-1}\right|\right] \\
& +\beta_{n}\left\|S_{n}-S_{n-1}\right\|_{\infty, B}+\gamma_{n} .
\end{aligned}
$$

Thus, by using conditions (i), (v), $\sum_{n=1}^{\infty}\left(\alpha_{n-1}-\alpha_{n}\right)<\infty, \sum_{n=1}^{\infty}\left|\beta_{n-1}-\beta_{n}\right|<\infty$ and applying Lemma 2.4, we conclude that

$$
\lim _{n \rightarrow \infty}\left\|x_{n+1}-x_{n}\right\|=0
$$

Step 3. We claim $\lim _{n \rightarrow \infty}\left\|x_{n}-T_{i} x_{n}\right\|=0$ for all $i \in \mathbb{N}$.

Since $T_{i} x_{n} \in C$ for all $i \in \mathbb{N}$ and $\sum_{i=1}^{n}\left(\alpha_{i-1}-\alpha_{i}\right)+\alpha_{n}=1$, we get

$$
\sum_{i=1}^{n}\left(\alpha_{i-1}-\alpha_{i}\right) T_{i} x_{n}+\alpha_{n} z \in C \quad \text { for all } z \in C
$$

Noticing $x_{n+1}=P_{C}\left(u_{n}\right)$ and fixing $z \in \bigcap_{n=1}^{\infty} F\left(T_{n}\right)$, from (3.1) we have

$$
\begin{aligned}
& \sum_{i=1}^{n}\left(\alpha_{i-1}-\alpha_{i}\right)\left(x_{n}-T_{i} x_{n}\right) \\
& =P_{C}\left(u_{n}\right)+\left(1-\alpha_{n}\right) x_{n}-\left(\sum_{i=1}^{n}\left(\alpha_{i-1}-\alpha_{i}\right) T_{i} x_{n}+\alpha_{n} z\right) \\
& \quad+\alpha_{n} z-x_{n+1} \\
& \quad P_{C}\left(u_{n}\right)-P_{C}\left(\sum_{i=1}^{n}\left(\alpha_{i-1}-\alpha_{i}\right) T_{i} x_{n}+\alpha_{n} z\right) \\
& \quad+\left(1-\alpha_{n}\right)\left(x_{n}-x_{n+1}\right)+\alpha_{n}\left(z-x_{n+1}\right) .
\end{aligned}
$$


Hence,

$$
\begin{aligned}
& \sum_{i=1}^{n}\left(\alpha_{i-1}-\alpha_{i}\right)\left\langle x_{n}-T_{i} x_{n}, x_{n}-p\right\rangle \\
& =\left\langle P_{C}\left(u_{n}\right)-P_{C}\left(\sum_{i=1}^{n}\left(\alpha_{i-1}-\alpha_{i}\right) T_{i} x_{n}+\alpha_{n} z\right), x_{n}-p\right\rangle \\
& +\left\langle\left(1-\alpha_{n}\right)\left(x_{n}-x_{n+1}\right), x_{n}-p\right\rangle+\alpha_{n}\left\langle z-x_{n+1}, x_{n}-p\right\rangle \\
& \leq\left\|u_{n}-\sum_{i=1}^{n}\left(\alpha_{i-1}-\alpha_{i}\right) T_{i} x_{n}-\alpha_{n} z\right\|\left\|x_{n}-p\right\| \\
& +\left(1-\alpha_{n}\right)\left\|x_{n}-x_{n+1}\right\|\left\|x_{n}-p\right\|+\alpha_{n}\left\|z-x_{n+1}\right\|\left\|x_{n}-p\right\| \\
& =\left\|\alpha_{n}\left(f\left(x_{n}\right)-z\right)+\sum_{i=1}^{n}\left(\alpha_{i-1}-\alpha_{i}\right)\left(T_{i} y_{n}-T_{i} x_{n}\right)\right\|\left\|x_{n}-p\right\| \\
& +\left(1-\alpha_{n}\right)\left\|x_{n}-x_{n+1}\right\|\left\|x_{n}-p\right\|+\alpha_{n}\left\|z-x_{n+1}\right\|\left\|x_{n}-p\right\| \\
& \leq \alpha_{n}\left\|f\left(x_{n}\right)-z\right\|\left\|x_{n}-p\right\|+\sum_{i=1}^{n}\left(\alpha_{i-1}-\alpha_{i}\right)\left(\left\|y_{n}-x_{n}\right\|+a_{i}\right)\left\|x_{n}-p\right\| \\
& +\left(1-\alpha_{n}\right)\left\|x_{n}-x_{n+1}\right\|\left\|x_{n}-p\right\|+\alpha_{n}\left\|z-x_{n+1}\right\|\left\|x_{n}-p\right\| \\
& \leq \alpha_{n}\left\|f\left(x_{n}\right)-z\right\|\left\|x_{n}-p\right\|+\left(1-\alpha_{n}\right)\left\|y_{n}-x_{n}\right\|\left\|x_{n}-p\right\| \\
& +\left(1-\alpha_{n}\right)\left\|x_{n}-x_{n+1}\right\|\left\|x_{n}-p\right\| \\
& +\alpha_{n}\left\|z-x_{n+1}\right\|\left\|x_{n}-p\right\|+\gamma_{n}\left\|x_{n}-p\right\| \\
& \leq \alpha_{n}\left\|f\left(x_{n}\right)-z\right\|\left\|x_{n}-p\right\|+\left(1-\alpha_{n}\right) \beta_{n}\left\|S_{n} x_{n}-x_{n}\right\|\left\|x_{n}-p\right\| \\
& +\left(1-\alpha_{n}\right)\left\|x_{n}-x_{n+1}\right\|\left\|x_{n}-p\right\|+\alpha_{n}\left\|z-x_{n+1}\right\|\left\|x_{n}-p\right\|+\gamma_{n} R \\
& \leq\left(2 \alpha_{n}+\beta_{n}\right) M^{\prime}+\left(1-\alpha_{n}\right)\left\|x_{n}-x_{n+1}\right\| R+\gamma_{n} R,
\end{aligned}
$$

where $R$ is a positive constant such that $\left\|x_{n}-p\right\| \leq R$ for all $n \in \mathbb{N}$ and

$$
M^{\prime}=\sup _{n \in \mathbb{N}}\left\{\left\|f\left(x_{n}\right)-z\right\|\left\|x_{n}-p\right\|,\left\|S_{n} x_{n}-x_{n}\right\|\left\|x_{n}-p\right\|,\left\|z-x_{n+1}\right\|\left\|x_{n}-p\right\|\right\} .
$$

Set $\xi_{n}:=\frac{1}{2}\left(a_{n}+2\left\|x_{n}-p\right\|\right) a_{n}$. From Proposition 2.2, using (3.7), we obtain that

$$
\begin{aligned}
& \frac{1}{2} \sum_{i=1}^{n}\left(\alpha_{i-1}-\alpha_{i}\right)\left\|x_{n}-T_{i} x_{n}\right\|^{2} \\
& \quad \leq \sum_{i=1}^{n}\left(\alpha_{i-1}-\alpha_{i}\right)\left\langle x_{n}-T_{i} x_{n}, x_{n}-p\right\rangle+\xi_{n} \\
& \quad \leq\left(2 \alpha_{n}+\beta_{n}\right) M^{\prime}+\left(1-\alpha_{n}\right)\left\|x_{n}-x_{n+1}\right\| R+\gamma_{n} R .
\end{aligned}
$$

Using (3.6), condition (i), $\lim _{n \rightarrow \infty} \frac{\gamma_{n}}{\beta_{n}}=0$ and $\lim _{n \rightarrow \infty} \frac{\beta_{n}}{\alpha_{n}}=\tau \in(0, \infty)$, we have

$$
\lim _{n \rightarrow \infty} \sum_{i=1}^{n}\left(\alpha_{i-1}-\alpha_{i}\right)\left\|x_{n}-T_{i} x_{n}\right\|=0 .
$$


Since $\left(\alpha_{i-1}-\alpha_{i}\right)\left\|x_{n}-T_{i} x_{n}\right\| \leq \sum_{i=1}^{n}\left(\alpha_{i-1}-\alpha_{i}\right)\left\|x_{n}-T_{i} x_{n}\right\|$ for all $i \in \mathbb{N}$ and $\left\{\alpha_{n}\right\}$ is strictly decreasing, we have

$$
\lim _{n \rightarrow \infty}\left\|x_{n}-T_{i} x_{n}\right\|=0 \quad \text { for all } i \in \mathbb{N}
$$

Step 4. $\left\|y_{n}-T_{i} y_{n}\right\| \rightarrow 0$ as $n \rightarrow \infty$ for all $i \in \mathbb{N}$.

Noticing that $\lim _{n \rightarrow \infty} \frac{\beta_{n}}{\alpha_{n}}=\tau \in(0, \infty)$ and using condition (i), we have $\beta_{n} \rightarrow 0$ as $n \rightarrow \infty$. Therefore, we obtain that

$$
\left\|y_{n}-x_{n}\right\|=\beta_{n}\left\|S_{n} x_{n}-x_{n}\right\| \rightarrow 0 \quad \text { as } n \rightarrow \infty
$$

So that for all $i \in \mathbb{N}$, we have

$$
\left\|y_{n}-T_{i} x_{n}\right\| \leq\left\|y_{n}-x_{n}\right\|+\left\|x_{n}-T_{i} x_{n}\right\| \rightarrow 0 \quad \text { as } n \rightarrow \infty \text {. }
$$

Since each $T_{i}$ is uniformly continuous, from (3.8) and (3.9), we have

$$
\left\|y_{n}-T_{i} y_{n}\right\| \leq\left\|y_{n}-T_{i} x_{n}\right\|+\left\|T_{i} x_{n}-T_{i} y_{n}\right\| \rightarrow 0 \text { as } n \rightarrow \infty
$$

for all $i \in \mathbb{N}$.

Step 5. $\lim _{n \rightarrow \infty} \frac{\left\|x_{n+1}-x_{n}\right\|}{\beta_{n}}=0$ and $\lim _{n \rightarrow \infty} \frac{\left\|u_{n}-u_{n-1}\right\|}{\beta_{n}}=\frac{\left\|u_{n}-u_{n-1}\right\|}{\alpha_{n}}=0$.

From (3.5) we obtain that

$$
\begin{aligned}
& \frac{\left\|x_{n+1}-x_{n}\right\|}{\beta_{n}} \\
& \leq \frac{\left\|u_{n}-u_{n-1}\right\|}{\beta_{n}} \\
& \leq\left(1-\alpha_{n}(1-\lambda)\right) \frac{\left\|x_{n}-x_{n-1}\right\|}{\beta_{n}} \\
& \quad+M\left(\frac{\left(\alpha_{n-1}-\alpha_{n}\right)}{\beta_{n}}+\frac{\left|\beta_{n}-\beta_{n-1}\right|}{\beta_{n}}\right)+\left\|S_{n}-S_{n-1}\right\|_{\infty, B}+\frac{\gamma_{n}}{\beta_{n}} \\
& =\left(1-\alpha_{n}(1-\lambda)\right) \frac{\left\|x_{n}-x_{n-1}\right\|}{\beta_{n-1}}+\left(1-\alpha_{n}(1-\lambda)\right)\left\|x_{n}-x_{n-1}\right\|\left(\frac{1}{\beta_{n}}-\frac{1}{\beta_{n-1}}\right) \\
& \quad+M\left(\frac{\left(\alpha_{n-1}-\alpha_{n}\right)}{\beta_{n}}+\frac{\left|\beta_{n}-\beta_{n-1}\right|}{\beta_{n}}\right)+\left\|S_{n}-S_{n-1}\right\|_{\infty, B}+\frac{\gamma_{n}}{\beta_{n}} .
\end{aligned}
$$

We observe that

$$
\left(1-\alpha_{n}(1-\lambda)\right)\left(\frac{1}{\beta_{n}}-\frac{1}{\beta_{n-1}}\right) \leq \alpha_{n} \frac{1}{\alpha_{n}}\left|\frac{1}{\beta_{n}}-\frac{1}{\beta_{n-1}}\right| \leq \alpha_{n} N
$$

Set

$$
\begin{aligned}
& \mu_{n}=\alpha_{n}(1-\lambda), \\
& \varphi_{n}=\alpha_{n} N\left\|x_{n}-x_{n-1}\right\|+M\left(\frac{\left(\alpha_{n-1}-\alpha_{n}\right)}{\beta_{n}}+\frac{\left|\beta_{n}-\beta_{n-1}\right|}{\beta_{n}}\right), \\
& v_{n}=\left\|S_{n}-S_{n-1}\right\|_{\infty, B}+\frac{\gamma_{n}}{\beta_{n}} .
\end{aligned}
$$


From (3.10) we obtain that

$$
\begin{aligned}
\frac{\left\|x_{n+1}-x_{n}\right\|}{\beta_{n}} \leq & \frac{\left\|u_{n}-u_{n-1}\right\|}{\beta_{n}} \\
\leq & \left(1-\alpha_{n}(1-\lambda)\right) \frac{\left\|x_{n}-x_{n-1}\right\|}{\beta_{n-1}}+\alpha_{n} N\left\|x_{n}-x_{n-1}\right\| \\
& +M\left(\frac{\left(\alpha_{n-1}-\alpha_{n}\right)}{\beta_{n}}+\frac{\left|\beta_{n}-\beta_{n-1}\right|}{\beta_{n}}\right)+\left\|S_{n}-S_{n-1}\right\|_{\infty, B}+\frac{\gamma_{n}}{\beta_{n}} \\
\leq & \left(1-\alpha_{n}(1-\lambda)\right) \frac{\left\|u_{n-1}-u_{n-2}\right\|}{\beta_{n-1}}+\alpha_{n} N\left\|x_{n}-x_{n-1}\right\| \\
& +M\left(\frac{\left(\alpha_{n-1}-\alpha_{n}\right)}{\beta_{n}}+\frac{\left|\beta_{n}-\beta_{n-1}\right|}{\beta_{n}}\right)+\left\|S_{n}-S_{n-1}\right\|_{\infty, B}+\frac{\gamma_{n}}{\beta_{n}} \\
\leq & \left(1-\mu_{n}\right) \frac{\left\|u_{n-1}-u_{n-2}\right\|}{\beta_{n-1}}+\varphi_{n}+v_{n} .
\end{aligned}
$$

Using conditions (i), (iii), (v) and applying Lemma 2.4, we have

$$
\lim _{n \rightarrow \infty} \frac{\left\|x_{n+1}-x_{n}\right\|}{\beta_{n}}=0 \text { and } \lim _{n \rightarrow \infty} \frac{\left\|u_{n}-u_{n-1}\right\|}{\beta_{n}}=\frac{\left\|u_{n}-u_{n-1}\right\|}{\alpha_{n}}=0 .
$$

Step 6. Set $v_{n}:=\frac{x_{n}-x_{n+1}}{\left(1-\alpha_{n}\right) \beta_{n}}$ and $u_{n}:=\alpha_{n} f\left(x_{n}\right)+\sum_{i=1}^{n}\left(\alpha_{i-1}-\alpha_{i}\right) T_{i} y_{n}$ for all $n \in \mathbb{N}$. Then, for any $z \in \bigcap_{n=1}^{\infty} F\left(T_{n}\right)$, we can calculate $\left\langle v_{n}, x_{n}-z\right\rangle$.

From (3.1) we have

$$
x_{n+1}=P_{C}\left(u_{n}\right)-u_{n}+\alpha_{n} f\left(x_{n}\right)+\sum_{i=1}^{n}\left(\alpha_{i-1}-\alpha_{i}\right)\left(T_{i} y_{n}-y_{n}\right)+\left(1-\alpha_{n}\right) y_{n} \text {, }
$$

which gives that

$$
\begin{aligned}
x_{n}- & x_{n+1} \\
= & \left(1-\alpha_{n}\right) x_{n}+\alpha_{n} x_{n} \\
& -\left(P_{C}\left(u_{n}\right)-u_{n}+\alpha_{n} f\left(x_{n}\right)+\sum_{i=1}^{n}\left(\alpha_{i-1}-\alpha_{i}\right)\left(T_{i} y_{n}-y_{n}\right)+\left(1-\alpha_{n}\right) y_{n}\right) \\
= & \left(1-\alpha_{n}\right) \beta_{n}\left(x_{n}-S_{n} x_{n}\right)+\left(u_{n}-P_{C}\left(u_{n}\right)\right) \\
& +\sum_{i=1}^{n}\left(\alpha_{i-1}-\alpha_{i}\right)\left(y_{n}-T_{i} y_{n}\right)+\alpha_{n}\left(x_{n}-f\left(x_{n}\right)\right) .
\end{aligned}
$$

Then we conclude that

$$
\begin{aligned}
& \frac{x_{n}-x_{n+1}}{\left(1-\alpha_{n}\right) \beta_{n}} \\
& =x_{n}-S_{n} x_{n}+\frac{1}{\left(1-\alpha_{n}\right) \beta_{n}}\left(u_{n}-P_{C}\left(u_{n}\right)\right) \\
& \quad+\frac{1}{\left(1-\alpha_{n}\right) \beta_{n}} \sum_{i=1}^{n}\left(\alpha_{i-1}-\alpha_{i}\right)\left(y_{n}-T_{i} y_{n}\right)+\frac{\alpha_{n}}{\left(1-\alpha_{n}\right) \beta_{n}}\left(x_{n}-f\left(x_{n}\right)\right) .
\end{aligned}
$$


Noticing that $x_{n+1}=P_{C}\left(u_{n}\right)$. For any $z \in \bigcap_{n=1}^{\infty} F\left(T_{n}\right)$, we have

$$
\begin{aligned}
\left\langle v_{n}, x_{n}-z\right\rangle & \\
= & \frac{1}{\left(1-\alpha_{n}\right) \beta_{n}}\left\langle u_{n}-P_{C}\left(u_{n}\right), P_{C}\left(u_{n-1}\right)-z\right\rangle+\left\langle x_{n}-S_{n} x_{n}, x_{n}-z\right\rangle \\
& +\frac{1}{\left(1-\alpha_{n}\right) \beta_{n}} \sum_{i=1}^{n}\left(\alpha_{i-1}-\alpha_{i}\right)\left\langle y_{n}-T_{i} y_{n}, x_{n}-z\right\rangle \\
& +\frac{\alpha_{n}}{\left(1-\alpha_{n}\right) \beta_{n}}\left\langle(I-f) x_{n}, x_{n}-z\right\rangle .
\end{aligned}
$$

By using Lemma 2.2, we obtain that

$$
\begin{aligned}
& \left\langle x_{n}-S_{n} x_{n}, x_{n}-z\right\rangle \\
& \quad=\left\langle\left(I-S_{n}\right) x_{n}-\left(I-S_{n}\right) z, x_{n}-z\right\rangle+\left\langle\left(I-S_{n}\right) z, x_{n}-z\right\rangle \\
& \quad \geq\left\langle\left(I-S_{n}\right) z, x_{n}-z\right\rangle, \\
& \left\langle(I-f) x_{n}, x_{n}-z\right\rangle \\
& \quad=\left\langle(I-f) x_{n}-(I-f) z, x_{n}-z\right\rangle+\left\langle(I-f) z, x_{n}-z\right\rangle \\
& \quad \geq(1-\lambda)\left\|x_{n}-z\right\|^{2}+\left\langle(I-f) z, x_{n}-z\right\rangle, \\
& \left\langle y_{n}-T_{i} y_{n}, x_{n}-z\right\rangle \\
& \quad=\left\langle\left(I-T_{i}\right) y_{n}-\left(I-T_{i}\right) z, x_{n}-y_{n}\right\rangle+\left\langle\left(I-T_{i}\right) y_{n}-\left(I-T_{i}\right) z, y_{n}-z\right\rangle \\
& \quad \geq\left\langle\left(I-T_{i}\right) y_{n}-\left(I-T_{i}\right) z, x_{n}-y_{n}\right\rangle-a_{i}\left\|y_{n}-z\right\| \\
& \quad=\beta_{n}\left\langle\left(I-T_{i}\right) y_{n}, x_{n}-S_{n} x_{n}\right\rangle-a_{i}\left\|y_{n}-z\right\| \quad \text { for all } i \in \mathbb{N} .
\end{aligned}
$$

Also, from Lemma 2.1 we get that

$$
\begin{aligned}
\left\langle u_{n}-P_{C}\left(u_{n}\right), P_{C}\left(u_{n-1}\right)-z\right\rangle= & \left\langle u_{n}-P_{C}\left(u_{n}\right), P_{C}\left(u_{n-1}\right)-P_{C}\left(u_{n}\right)\right\rangle \\
& +\left\langle u_{n}-P_{C}\left(u_{n}\right), P_{C}\left(u_{n}\right)-z\right\rangle \\
\geq & \left\langle u_{n}-P_{C}\left(u_{n}\right), P_{C}\left(u_{n-1}\right)-P_{C}\left(u_{n}\right)\right\rangle .
\end{aligned}
$$

Substituting (3.12), (3.13), (3.14) and (3.15) into (3.11), we have

$$
\begin{aligned}
\left\langle v_{n}, x_{n}-z\right\rangle & \\
\geq & \frac{1}{\left(1-\alpha_{n}\right) \beta_{n}}\left\langle u_{n}-P_{C}\left(u_{n}\right), P_{C}\left(u_{n-1}\right)-P_{C}\left(u_{n}\right)\right\rangle+\left\langle\left(I-S_{n}\right) z, x_{n}-z\right\rangle \\
& +\frac{1}{\left(1-\alpha_{n}\right)} \sum_{i=1}^{n}\left(\alpha_{i-1}-\alpha_{i}\right)\left[\left\langle\left(I-T_{i}\right) y_{n}, x_{n}-S_{n} x_{n}\right\rangle-a_{i}\left\|y_{n}-z\right\|\right] \\
& +\frac{\alpha_{n}}{\left(1-\alpha_{n}\right) \beta_{n}}\left\langle(I-f) z, x_{n}-z\right\rangle+\frac{\alpha_{n}(1-\lambda)}{\left(1-\alpha_{n}\right) \beta_{n}}\left\|x_{n}-z\right\|^{2} .
\end{aligned}
$$

Step 7. $x_{n} \rightarrow \tilde{x}$, where $\tilde{x}$ is a strong cluster point of the sequence $\left\{x_{n}\right\}$. 
From (3.16) we observe that

$$
\begin{aligned}
\left\|x_{n}-z\right\|^{2} \leq & \frac{\left(1-\alpha_{n}\right) \beta_{n}}{\alpha_{n}(1-\lambda)}\left[\left\langle v_{n}, x_{n}-z\right\rangle-\left\langle\left(I-S_{n}\right) z, x_{n}-z\right\rangle\right] \\
& -\frac{1}{1-\lambda}\left\langle(I-f) z, x_{n}-z\right\rangle \\
& -\frac{\beta_{n}}{\alpha_{n}(1-\lambda)} \sum_{i=1}^{n}\left(\alpha_{i-1}-\alpha_{i}\right)\left[\left\langle\left(I-T_{i}\right) y_{n}, x_{n}-S_{n} x_{n}\right\rangle-a_{i}\left\|y_{n}-z\right\|\right] \\
& +\frac{\left\|u_{n}-u_{n-1}\right\|}{\alpha_{n}(1-\lambda)}\left\|u_{n}-P_{C}\left(u_{n}\right)\right\| .
\end{aligned}
$$

Noticing that $v_{n}=\frac{x_{n}-x_{n+1}}{\left(1-\alpha_{n}\right) \beta_{n}} \rightarrow 0, \frac{\left\|u_{n}-u_{n-1}\right\|}{\alpha_{n}} \rightarrow 0$, and for all $i \in \mathbb{N},\left(I-T_{i}\right) y_{n} \rightarrow 0$ as $n \rightarrow \infty$. It is easy to see that a weak cluster point of $\left\{x_{n}\right\}$ is also a strong cluster point. Note that the sequence $\left\{x_{n}\right\}$ is bounded, then there exists a subsequence $\left\{x_{n_{k}}\right\}$ of $\left\{x_{n}\right\}$ which converges to a point $x^{*} \in C$. We also have, for all $i \in \mathbb{N},\left(I-T_{i}\right) x_{n} \rightarrow 0$ as $n \rightarrow \infty$. By using condition (G), we get that $x^{*} \in F(T)$. Thus, for all $z \in \bigcap_{n=1}^{\infty} F\left(T_{n}\right)$, we obtain that

$$
\begin{aligned}
& \left\langle(I-f) x_{n_{k}}, x_{n_{k}}-z\right\rangle \\
& \leq \frac{\left(1-\alpha_{n_{k}}\right) \beta_{n_{k}}}{\alpha_{n_{k}}}\left\langle v_{n_{k}}, x_{n_{k}}-z\right\rangle-\frac{1}{\alpha_{n_{k}}}\left\|u_{n_{k}}-P_{C}\left(u_{n_{k}}\right)\right\|\left\|u_{n_{k}-1}-u_{n_{k}}\right\| \\
& \quad-\frac{\left(1-\alpha_{n_{k}}\right) \beta_{n_{k}}}{\alpha_{n_{k}}}\left\langle\left(I-S_{n_{k}}\right) z, x_{n_{k}}-z\right\rangle \\
& \quad-\frac{\beta_{n_{k}}}{\alpha_{n_{k}}} \sum_{i=1}^{n_{k}}\left(\alpha_{i-1}-\alpha_{i}\right)\left\langle\left(I-T_{i}\right) y_{n_{k}}, x_{n_{k}}-S_{n_{k}} x_{n_{k}}\right\rangle+\frac{\beta_{n_{k}} \gamma_{n_{k}}}{\alpha_{n_{k}}}\left\|y_{n_{k}}-z\right\| .
\end{aligned}
$$

Now, letting $k \rightarrow \infty$, we have

$$
\left\langle(I-f) x^{*}, x^{*}-z\right\rangle \leq-\tau\left\langle(I-S) z, x^{*}-z\right\rangle \quad \text { for all } z \in \bigcap_{n=1}^{\infty} F\left(T_{n}\right) .
$$

Now, since variational inequality problem (3.2) has the unique solution, then we get that $\omega_{w}\left(x_{n}\right)=\{\tilde{x}\}$. Note that every weak cluster point of the sequence $\left\{x_{n}\right\}$ is also a strong cluster point. Then we have $\lim _{n \rightarrow \infty} x_{n}=\tilde{x}$.

Recently, Marino et al. [28] used a different approach to obtain the convergence of a more general iterative method that involves an equilibrium problem. We now present the result of Gu et al. [18, Theorem 3.5] as a corollary.

Corollary 3.1 Let $C$ be a nonempty closed convex subset of a real Hilbert space $H$. Let $f: C \rightarrow H$ be a $\lambda$-contraction. Let $S: C \rightarrow C$ be a nonexpansive mapping, and let $\mathcal{T}=$ $\left\{T_{n}\right\}_{n=1}^{\infty}$ be a countable family of nonexpansive mappings from $C$ into itself such that $F(\mathcal{T})=$ $\bigcap_{n=1}^{\infty} F\left(T_{n}\right) \neq \emptyset$. For arbitrary $x_{1} \in C$, consider the sequence $\left\{x_{n}\right\}$ generated by the following iterative process:

$$
\left\{\begin{array}{l}
x_{1} \in C, \\
y_{n}=\beta_{n} S x_{n}+\left(1-\beta_{n}\right) x_{n}, \\
x_{n+1}=P_{C}\left[\alpha_{n} f\left(x_{n}\right)+\sum_{i=1}^{n}\left(\alpha_{i-1}-\alpha_{i}\right) T_{i} y_{n}\right]
\end{array}\right.
$$


for all $n \in \mathbb{N}$, where $\alpha_{0}=1,\left\{\alpha_{n}\right\}$ is a strictly decreasing sequence in $(0,1)$ and $\left\{\beta_{n}\right\}$ is a sequence in $(0,1)$ satisfying conditions (i)-(iv) of Theorem 3.1. Then the sequence $\left\{x_{n}\right\}$ converges strongly to a point $x^{*} \in \bigcap_{n=1}^{\infty} F\left(T_{n}\right)$, which is the unique solution of the following variational inequality:

$$
\left\langle\frac{1}{\tau}(I-f) x^{*}+(I-S) x^{*}, x-x^{*}\right\rangle \geq 0 \quad \text { for all } x \in \bigcap_{n=1}^{\infty} F\left(T_{n}\right) .
$$

Again, we present the result of Yao et al. [17, Theorem 3.2] as a corollary.

Corollary 3.2 Let $C$ be a nonempty closed convex subset of a real Hilbert space H. Let $f: C \rightarrow H$ be a $\lambda$-contraction. Let $S: C \rightarrow C$ be a nonexpansive mapping, and let $T: C \rightarrow C$ be a nonexpansive mapping such that $F(T) \neq \emptyset$. For arbitrary $x_{1} \in C$, consider the sequence $\left\{x_{n}\right\}$ generated by the following iterative process:

$$
\left\{\begin{array}{l}
x_{1} \in C \\
y_{n}=\left(1-\beta_{n}\right) x_{n}+\beta_{n} S x_{n} \\
x_{n+1}=P_{C}\left[\alpha_{n} f\left(x_{n}\right)+\left(1-\alpha_{n}\right) T y_{n}\right]
\end{array}\right.
$$

for all $n \in \mathbb{N}$, where $\left\{\alpha_{n}\right\}$ and $\left\{\beta_{n}\right\}$ are two sequences in $(0,1)$ satisfying conditions (i)-(iv) of Theorem 3.1. Then the sequence $\left\{x_{n}\right\}$ converges strongly to a point $x^{*} \in F(T)$, which is the unique solution of the following variational inequality:

$$
\left\langle\frac{1}{\tau}(I-f) x^{*}+(I-S) x^{*}, x-x^{*}\right\rangle \geq 0 \quad \text { for all } x \in F(T) .
$$

Theorem 3.2 Let $C$ be a nonempty closed convex subset of a real Hilbert space H. Let $f: C \rightarrow H$ be a $\lambda$-contraction, and let $\left\{S_{n}\right\}$ be a sequence of nonexpansive mappings from $C$ into itself. Let $S$ be a nonexpansive mapping from C into itself such that $\lim _{n \rightarrow \infty} S_{n} x=S x$ for all $x \in C$. Let $t_{1}, t_{2}, t_{3}, \ldots, t_{N}>0$ such that $\sum_{i=1}^{N} t_{i}=1$. Let $T_{1}, T_{2}, T_{3}, \ldots, T_{N}: C \rightarrow C$ be nonexpansive mappings such that $\bigcap_{i=1}^{N} F\left(T_{i}\right) \neq \emptyset$, and assume that $T=\sum_{i=1}^{N} t_{i} T_{i}$. For arbitrary $x_{1} \in C$, consider the sequence $\left\{x_{n}\right\}$ generated by the following iterative process:

$$
\left\{\begin{array}{l}
x_{1} \in C \\
y_{n}=\beta_{n} S_{n} x_{n}+\left(1-\beta_{n}\right) x_{n} \\
x_{n+1}=P_{C}\left[\alpha_{n} f\left(x_{n}\right)+\left(1-\alpha_{n}\right) \sum_{i=1}^{N} t_{i} T_{i} y_{n}\right]
\end{array}\right.
$$

for all $n \in \mathbb{N}$, where $\left\{\alpha_{n}\right\}$ and $\left\{\beta_{n}\right\}$ are two sequences in $(0,1)$ satisfying conditions (i)-(iv) of Theorem 3.1. Then the sequence $\left\{x_{n}\right\}$ converges strongly to a point $x^{*} \in \bigcap_{i=1}^{N} F\left(T_{i}\right)$, which is the unique solution of the following variational inequality:

$$
\left\langle\frac{1}{\tau}(I-f) x^{*}+(I-S) x^{*}, x-x^{*}\right\rangle \geq 0 \quad \text { for all } x \in \bigcap_{i=1}^{N} F\left(T_{i}\right) .
$$

Proof The proof follows from Lemma 2.5 and Corollary 3.2. 


\section{Numerical example}

We present an example to show the effectiveness and convergence of the sequence generated by the considered iterative scheme.

Example 4.1 Let $H=\mathbb{R}$ and $C=[0,1]$. Let $T$ be a self-mapping defined by $T x=1-x$ for all $x \in C$. Let $f: C \rightarrow H$ be a contraction mapping defined by $f(x)=\frac{2}{3} x-\frac{1}{6}$ for all $x \in C$, and let $\left\{S_{n}\right\}$ be a sequence of nonexpansive mappings from $C$ into itself defined by $S_{n} x=x+\frac{1}{2 n}$ such that $S x=\lim _{n \rightarrow \infty} S_{n} x$ for all $x \in C$, where $S$ is a nonexpansive mapping defined by $S x=x$ for all $x \in C$. Define sequences $\left\{\alpha_{n}\right\}$ and $\left\{\beta_{n}\right\}$ in $(0,1)$ by $\alpha_{n}=\beta_{n}=\frac{1}{(n+1)^{1 / 2}}$. Without loss of generality, we may assume that $a_{n}=\frac{1}{n^{3 / 2}}$ for all $n \in \mathbb{N}$. For each $n \in \mathbb{N}$, define $T_{n}: C \rightarrow C$ by

$$
T_{n} x= \begin{cases}1-x & \text { if } x \in[0,1), \\ a_{n} & \text { if } x=1 .\end{cases}
$$

It was shown in [19] that $\mathcal{T}=\left\{T_{n}\right\}$ is a sequence of nearly nonexpansive mappings from $C$ into itself such that $F(\mathcal{T})=\left\{\frac{1}{2}\right\}$ and $T x=\lim _{n \rightarrow \infty} T_{n} x$ for all $x \in C$, where $T$ is a nonexpansive mapping.

One can observe that all the assumptions of Theorem 3.1 are satisfied, and the sequence $\left\{x_{n}\right\}$ generated by (3.1) converges to a unique solution $\frac{1}{2}$ of variational inequality (3.2) over $F(\mathcal{T})$.

\section{Competing interests}

The authors declare that they have no competing interests.

\section{Authors' contributions}

All authors read and approved the final manuscript.

\section{Author details}

${ }^{1}$ Department of Mathematics, Banaras Hindu University, Varanasi, 221005, India. ${ }^{2}$ Department of Mathematics and RINS Gyeongsang National University, Jinju, 660-701, Korea.

\section{Acknowledgements}

The authors would like to thank the editor and referees for useful comments and suggestions.

Received: 10 June 2013 Accepted: 10 October 2013 Published: \#PUBLICATION_DATE

\section{References}

1. Stampacchia, G: Formes bilineaires coercivities sur les ensembles convexes. C. R. Acad. Sci. Paris $258,4413-4416$ (1964)

2. Censor, Y, lusem, AN, Zenios, SA: An interior point method with Bregman functions for the variational inequality problem with paramonotone operators. Math. Program. 81(3), 373-400 (1998). doi:10.1007/BF01580089

3. Shi, P: Equivalence of variational inequalities with Wiener-Hopf equations. Proc. Am. Math. Soc. 111(2), 339-346 (1991). doi:10.2307/2048322

4. Xu, HK, Kim, TH: Convergence of hybrid steepest-descend methods for variational inequalities. J. Optim. Theory Appl. 119(1), 185-201 (2003). doi:10.1023/B:JOTA.0000005048.79379.b6

5. Yao, JC: Variational inequalities with generalized monotone operators. Math. Oper. Res. 19(3), 691-705 (1994) doi:10.1287/moor.19.3.691

6. Baiocchi, C, Capelo, A: Variational and Quasivariational Inequalities: Applications to Free Boundary Problems. Wiley, New York (1984)

7. Ekeland, I, Témam, R: Convex Analysis and Variational Problems. Classics in Applied Mathematics. SIAM, Philadelphia (1999)

8. Facchinei, F, Pang, JS: Finite Dimensional Variational Inequalities and Complementarity Problems I. Springer, New York (2003)

9. Kikuchi, N, Oden, JT: Contact Problems in Elasticity: A Study of Variational Inequalities and Finite Element Methods. SIAM Studies in Applied Mathematics. SIAM, Philadelphia (1988)

10. Kinderlehrer, D, Stampacchia, G: An Introduction to Variational Inequalities and Their Applications. Academic Press, New York (1980) 
11. Takahashi, W: Nonlinear Functional Analysis. Yokohama Publishers, Yokohama (2000)

12. Moudafi, A, Mainge, PE: Towards viscosity approximations of hierarchical fixed points problems. Fixed Point Theory Appl. 2006, Article ID 95453 (2006). doi:10.1155/FPTA/2006/95453

13. Cianciaruso, F, Marino, G, Muglia, L, Yao, Y: On a two step algorithm for hierarchical fixed point problems and variational inequalities. J. Inequal. Appl. 2009, Article ID 208692 (2009). doi:10.1155/2009/208692

14. Maingé, PE, Moudafi, A: Strong convergence of an iterative method for hierarchical fixed point problems. Pac. J. Optim. 3(3), 529-538 (2007)

15. Marino, G, Xu, HK: Explicit hierarchical fixed point approach to variational inequalities. J. Optim. Theory Appl. 149(1), 61-78 (2011). doi:10.1007/s10957-010-9775-1

16. Moudafi, A: Krasnoselski-Mann iteration for hierarchical fixed points problems. Inverse Probl. 23(4), 1635-1640 (2007). doi:10.1088/0266-5611/23/4/015

17. Yao, Y, Cho, YJ, Liou, YC: Iterative algorithms for hierarchical fixed point problems and variational inequalities. Math. Comput. Model. 52(9-10), 1697-1705 (2010). doi:10.1016/j.mcm.2010.06.038

18. Gu, G, Wang, S, Cho, YJ: Strong convergence algorithms for hierarchical fixed point problems and variational inequalities. J. Appl. Math. 2011, Article ID 164978 (2011). doi:10.1155/2011/164978

19. Sahu, DR, Wong, NC, Yao, JC: A generalized hybrid steepest-descent method for variational inequalities in Banach spaces. Fixed Point Theory Appl. 2011, Article ID 754702 (2011). doi:10.1155/2011/754702

20. Moudafi, A: Viscosity approximation methods for fixed points problems. J. Math. Anal. Appl. 241(1), 46-55 (2000). doi:10.1006/jmaa.1999.6615

21. Xu, HK: Viscosity approximation methods for nonexpansive mappings. J. Math. Anal. Appl. 298(1), 279-291 (2004) doi:10.1016/j.jmaa.2004.04.059

22. Agarwal, RP, O'Regan, D, Sahu, DR: Fixed Point Theory for Lipschitzian-Type Mappings with Applications. Topological Fixed Point Theory and Its Applications. Springer, New York (2009)

23. Sahu, DR: Fixed points of demicontinuous nearly Lipschitzian mappings in Banach spaces. Comment. Math. Univ. Carol. 46(4), 653-666 (2005)

24. Wong, NC, Sahu, DR, Yao, JC: Solving variational inequalities involving nonexpansive type mappings. Nonlinear Anal. 69(12), 4732-4753 (2008). doi:10.1016/j.na.2007.11.025

25. Marino, G, Xu, HK: A general iterative method for nonexpansive mappings in Hilbert spaces. J. Math. Anal. Appl. 318(1), 43-52 (2006). doi:10.1016/j.jmaa.2005.05.028

26. Maingé, PE: Approximation methods for common fixed points of nonexpansive mappings in Hilbert spaces. J. Math. Anal. Appl. 325(1), 469-479 (2007). doi:10.1016/j.jmaa.2005.12.066

27. Bruck, RE: Properties of fixed point sets of nonexpansive mapping in Banach spaces. Trans. Am. Math. Soc. 179, 251-262 (1973). doi:10.2307/1996502

28. Marino, G, Muglia, L, Yao, Y: Viscosity methods for common solutions for equilibrium and variational inequality problems via multi step iterative algorithms and common fixed points. Nonlinear Anal. 75(4), 1787-1798 (2012) doi:10.1016/j.na.2011.09.019

\#DIGITAL_OBJECT_IDENTIFIER

Cite this article as: Sahu et al.: Iterative methods for hierarchical common fixed point problems and variational inequalities. Fixed Point Theory and Applications \#CITATION

\section{Submit your manuscript to a SpringerOpen ${ }^{\circ}$ journal and benefit from:}

- Convenient online submission

Rigorous peer review

- Immediate publication on acceptance

- Open access: articles freely available online

- High visibility within the field

- Retaining the copyright to your article 